\title{
Unravelling intimacies between plants and their enemies
}

All organisms live in close associations with other organisms. As we will recognise from our own relationships with others, partners can be perceived differently, sometimes with friendliness but other times with hostility. Plant encounters with other organisms, which can negatively or positively affect performance of the plant, are very complex as well, and this has fascinated plant scientists for more than a century. The economic and social consequences of intimate encounters of crop plants with harmful pathogens and insects are enormous, as exemplified by the high annual crop losses $(25-40 \%)$ due to plant diseases and pests, representing a value of over $€ 450$ billion worldwide. As a result of the rapidly growing human population, mankind faces the enormous challenge of increasing agricultural productivity while decreasing our ecological footprint in a changing climate. Hence, novel strategies for crop protection need to be developed, which creates major challenges for the plant science research community. This special issue of Plant Biology demonstrates that research on plantattacker interactions now encompasses highly diverse approaches, from in silico genome-wide analyses to laboratory and field tests, all concentrating on the question of how the plant immune system orchestrates the intimacies with different interacting organisms.

The rapid development of next-generation sequencing technology and of the associated bioinformatics needed for the assembly of sequence reads has resulted in the generation of genome sequences for numerous plants and their interacting organisms. The review of Hayward et al. (2012) reports that by studying the co-evolution of resistance genes of the Brassica species canola, and virulence genes of its fungal pathogen Leptosphaeria maculans (the causal agent of blackleg disease), novel insights in the interaction between these two organisms have been gained. Using this methodology, a wealth of information has been provided, which will aid in development of new crop protection strategies for this and other important agro-economic diseases.

A very different approach to unravel plant immunity is to relate different properties of a plant to its level of resistance to a specific disease. Besides the genetic properties, it is also possible to study the properties of defence responses. El Chartouni et al. (2012) investigated wheat cultivars having different levels of resistance/tolerance to the important pathogen Mycosphaerella graminicola. These authors examined cytological parameters, e.g. formation of mesophyll necrosis, and biochemical parameters, e.g. peroxidase activity. Although no direct correlation between any single parameter and the resistance level of the plants could be identified, hierarchical multi-factorial analysis revealed that specific correlations between cytological and biochemical data correspond to the different levels of resistance among the cultivars.
Maulik et al. (2012) followed a genetic bioinformatics approach to investigate the evolution of a class of defence proteins, the so-called polygalacturonase-inhibiting proteins (PGIPs), which inhibit plant cell wall-degrading fungal polygalacturonidases and thereby prevent pathogen invasion. They report that two of the ten tandemly-arranged leucinerich repeats (LRRs) of PGIPs are enriched in mutations and form separate clusters in phylogenetic trees, both within and across plant species. This diversification of PGIPs likely reflects their adaptive significance in recognising different pathogens. Ferrari et al. (2012) illustrate the impact of PGIPs on plant resistance. Fusarium graminearum, the causal agent of Fusarium head blight on wheat, infects the anthers of diverse plant species, and these authors show that overexpression of PGIPs protects flowers of wheat and Arabidopsis thaliana against infection by this fungus. Potentially, in agricultural practice, the yield of wheat could be improved and mycotoxin accumulation prevented.

Many responses are induced in plants upon encountering pathogens but relatively few of them have been characterised. Brauc et al. (2012) investigated the effect of the pathogeninduced gene encoding arginase $(A R G A H)$, which catalyses catabolism of the amino acid arginine into ornithine, a precursor of proline. Transgenic ARGAH overexpression in Arabidopsis lines caused enhanced resistance to the necrotrophic fungal pathogen Botrytis cinerea, which was correlated with enhanced levels of proline, a scavenger of reactive oxygen species and building block for cell wall reinforcement. Zhang et al. (2012) investigated a relatively well-studied defence component, namely the transcriptional regulator NPR1, which they isolated from Malus hupehensis, an apple species with high resistance to fungal diseases that affect commercial apple cultivars. Overexpression of MhNPR1 in tobacco plants led to enhanced expression of well-known defence-related $P R$ (pathogenesis-related) genes and improved resistance to B. cinerea.

Plants release volatile organic compounds (VOCs) that can attract natural enemies of pest insects or induce intact neighbouring plants to activate their defences. The plant hormone jasmonic acid (JA) is implicated in the production of VOCs, of which half are terpenoids. Semiz et al. (2012) compared JA-induced VOC emission profiles in forest tree seedlings that have different terpene-storing capacities. Scots pine (Pinus sylvestris) and holm oak (Quercus ilex) responded differentially in terms of mono-terpene emissions, but both tree species showed increased levels of sesquiterpene and green leaf volatiles after JA treatment. Blanch et al. (2012) studied the effect of phosphorus availability in Pinus pinaster seedlings on terpene content and emission. The theory of carbon nutrient balance states that when growth is limited by nutrients, plants allocate any excess carbon to the production of secondary metabolites. Indeed, phosphate stress was shown 
to lead to increased levels of foliar terpenes, while emission levels of terpenes were associatively reduced. In contrast, phosphorus-rich conditions, as found in tree nurseries, not only enhanced growth but also enhanced emission of specific monoterpenes, and this was especially the case in Pinus genotypes that are susceptible to the herbivorous pine weevil Hylobius abietis, which is attracted to these compounds.

In nature and agriculture, plants live in complex environments in which they encounter simultaneously or sequentially a multitude of pathogens, insects and beneficial microorganisms. Local interaction with a microbe or insect can activate systemic defences in plant tissues, thereby affecting the level of resistance to diverse subsequent attackers. Moreover, in order to establish an intimate relationship with the plant, interacting organisms have evolved many ways to suppress the plant immune system. Kyndt et al. (2012) studied the effect of different types of parasitic root nematode on systemic defence-related gene expression in rice. They found that the nematodes were capable of repressing various defence responses. Pineda et al. (2012) report on the effects of colonisation of the rhizosphere by beneficial Pseudomonas fluorescens bacteria. Generally, this leads to an improvement in the level of resistance against various attackers in systemic tissues; however, these authors describe an adverse effect on the resistance level of Arabidopsis to the phloem-sucking aphid Myzus persicae. This negative effect was correlated with repression and priming of different aphid-induced transcriptional responses.

The papers included here underscore the complexity of the immune signalling network that is steered by both the plant and its attackers. Undoubtedly, knowledge generated from research such as described in this special issue of Plant Biology will open new avenues for the engineering of durable resistance to pathogens and pests in crop plants.

Saskia C. M. van Wees Plant-Microbe Interactions, Department of Biology, Faculty of Science, Utrecht University, PO Box 800.56, 3508 TB Utrecht, The Netherlands E-mail:s.vanwees@uu.nl

\section{References}

Blanch J.-S., Sampedro L., Llusià J., Moreira X., Zas R., Peñuelas J. (2012) Effects of phosphorus availability and genetic variation of leaf terpene content and emission rate in Pinus pinaster seedlings susceptible and resistant to the pine weevil, Hylobius abietis. Plant Biology, 14 (Suppl. 1), 6672.

Brauc S., De Vooght E., Claeys M., Geuns J.M.C., Höfte M., Angenon G. (2012) Overexpression of arginase in Arabidopsis thaliana influences defence responses against Botrytis cinerea. Plant Biology, 14 (Suppl. 1), 39-45.

El Chartouni L., Randoux B., Duyme F., Renard-Merlier D., Tisserant B., Bourdon N., Pillon V., Sanssené J., Durand R., Reignault P., Halama P. (2012) Correlation of cytological and biochemical parameters with resistance and tolerance to Mycosphaerella graminicola in wheat. Plant Biology, 14 (Suppl. 1), $11-21$

Ferrari S., Sella L., Janni M., De Lorenzo G., Favaron F., D'Ovidio R. (2012) Transgenic expression of polygalacturonase-inhibiting proteins in Arabidopsis and wheat increases resistance to the flower pathogen Fusarium graminearum. Plant Biology, 14 (Suppl. 1), 31-38.

Hayward A., McLanders J., Campbell E., Edwards D., Batley J. (2012) Genomic advances will herald new insights into the Brassica:Leptosphaeria maculans pathosystem. Plant Biology, 14 (Suppl. 1), 1-10.

Kyndt T., Nahar K., Haegeman A., De Vleesschauwer D., Höfte M., Gheysen G. (2012) Comparing systemic defence-related gene expression changes upon migratory and sedentary nematode attack in rice. Plant Biology, 14 (Suppl. 1), 73-82.

Maulik A., Sarkar A.I., Devi S., Basu S. (2012) Polygalacturonase-inhibiting proteins - leucine-rich repeat proteins in plant defence. Plant Biology, 14 (Suppl. 1), 22-30.

Pineda A., Zheng S.-J., van Loon J.J.A., Dicke M. (2012) Rhizobacteria modify plant-aphid interactions: a case of induced systemic susceptibility. Plant Biology, 14 (Suppl. 1), 83-90.

Semiz G., Blande J.D., Heijari J., Issk K., Niinemets Ü., Holopainen J.K. (2012) Manipulation of VOC emissions with methyl jasmonate and carrageenan in the evergreen conifer Pinus sylvestris and evergreen broadleaf Quercus ilex. Plant Biology, 14 (Suppl. 1), 57-65.

Zhang J.-Y., Qiao Y.-S., Lv D., Gao Z.-H., Qu S.-C., Zhang Z. (2012) Malus hupehensis NPR1 induces pathogenesis-related protein gene expression in transgenic tobacco. Plant Biology, 14 (Suppl. 1), $46-56$. 